\title{
Functional characterization of Vif proteins from HIV-1 infected patients with different APOBEC3G haplotypes
}

\section{Kavidha Reddy ${ }^{\mathrm{a}, \mathrm{b}, *}$, Marcel Ooms ${ }^{\mathrm{c}, *}$, Michael Letko ${ }^{\mathrm{c}}$, Nigel Garrett ${ }^{\mathrm{d}}$, Viviana Simon ${ }^{c}$ and Thumbi Ndung' $\mathbf{u}^{\mathrm{a}, \mathrm{b}, \mathrm{e}, \mathrm{f}}$}

\begin{abstract}
Objective: The human cytidine deaminase APOBEC3G (A3G) potently restricts HIV-1 but the virus, in turn, expresses a Vif protein which degrades A3G. A natural A3GH186R variant, common in African populations, has been associated with a more rapid AIDS disease progression, but the underlying mechanism remains unknown. We hypothesized that differences in HIV-1 Vif activity towards A3G wild type and A3G-H186R contribute to the distinct clinical AIDS manifestation.
\end{abstract}

Methods: Vif variants were cloned from plasma samples of 26 South African HIV-1 subtype C infected patients, which either express wild type A3G or A3G-H186R. The Vif alleles were assessed for their ability to counteract $\mathrm{A} 3 \mathrm{G}$ variants using western blot and single-cycle infectivity assays.

Results: We obtained a total of 392 Vif sequences which displayed an amino acid sequence difference of $6.2-19.2 \%$ between patients. The intrapatient $V$ if diversities from patient groups $\mathrm{A} 3 \mathrm{G}^{\mathrm{WT} / \mathrm{WT}}, \mathrm{A} 3 \mathrm{G}^{\mathrm{WT} / \mathrm{H} 186 \mathrm{R}}$ and $\mathrm{A} 3 \mathrm{G}^{\mathrm{H} 186 \mathrm{R} / \mathrm{H} 186 \mathrm{R}}$ were similar. Vif variants obtained from patients expressing $A 3 G^{W T / N T}$ and $A 3 G^{H 186 R / H 186 R}$ were capable of counteracting both A3G variants with similar efficiency. However, the antiviral activity of A3G-H186R was significantly reduced in both the presence and absence of Vif, indicating that the A3G-H186R variant intrinsically exerts less antiviral activity.

Conclusion: A3G wild type and A3G-H186R are equally susceptible to counteraction by $V$ if, regardless of whether the $V$ if variant was obtained from $A 3 G^{W T / W T}$ and A3G $\mathrm{H}^{\mathrm{H} 186 \mathrm{R} / \mathrm{H} 186 \mathrm{R}}$ patients. However, the A3G-H186R variant intrinsically displayed lower antiviral activity, which could explain the higher plasma viral loads and accelerated disease progression reported for patients expressing $\mathrm{A}_{3} \mathrm{G}^{\mathrm{H} 186 \mathrm{R} / \mathrm{H} 186 \mathrm{R}}$.

Copyright (๑) 2016 Wolters Kluwer Health, Inc. All rights reserved.

AIDS 2016, 30:1723-1729

Keywords: APOBEC3, APOBEC3G, HIV, HIV-1, restriction factor, subtype C, Vif

\footnotetext{
${ }^{a}$ KwaZulu-Natal Research Institute for Tuberculosis and HIV (K-RITH), bHIV Pathogenesis Programme, Doris Duke Medical Research Institute, Nelson R. Mandela School of Medicine, University of KwaZulu-Natal, Durban, South Africa, ' Department of Microbiology and Global Health and Emerging Pathogens Institute, Icahn School of Medicine at Mount Sinai, New York, USA, ${ }^{\mathrm{d}}$ Centre for the AIDS Programme of Research in South Africa (CAPRISA), University of KwaZulu-Natal, Durban, South Africa, ${ }^{\mathrm{e}}$ Ragon Institute of MGH, MIT and Harvard University, Cambridge, Massachusetts, USA, and ${ }^{\mathrm{f}}$ Max Planck Institute for Infection Biology, Chariteplatz, Berlin, Germany.

Correspondence to Thumbi Ndung'u, PhD, KwaZulu-Natal Research Institute for Tuberculosis and HIV (K-RITH), K-RITH Tower Building, Level 5, University of KwaZulu-Natal, 719 Umbilo Road, Durban 4013, South Africa.

Tel: +27 31 2604733; fax: +27 31260 4623; e-mail: ndungu@ukzn.ac.za

* Kavidha Reddy and Marcel Ooms contributed equally to this work.

Received: 12 October 2015; revised: 29 March 2016; accepted: 6 April 2016.
} 


\section{Introduction}

Successful HIV-1 replication in the host depends on its ability to evade a myriad of innate and adaptive immune defences [1-7]. Among the innate immune factors that exert pressure on HIV-1 are host restriction factors such as the apolipoprotein B mRNA-editing enzyme catalytic polypeptide-like $3 \mathrm{G}$ protein (APOBEC 3G, A3G), which belongs to the family of cytidine deaminases $[8,9]$. A3G inhibits HIV-1 replication by deaminating single stranded viral DNA during reverse transcription, resulting in guanine-to-adenine mutations across the proviral genome $[8,10,11]$. Deaminase-independent mechanisms of $A 3 \mathrm{G}$ restriction have also been described [12-15]. HIV-1 viral infectivity factor protein, Vif, promotes the proteasomal degradation of $\mathrm{A} 3 \mathrm{G}$, allowing productive viral replication [16-18].

A natural $\mathrm{A} 3 \mathrm{G}$ polymorphism $\mathrm{H} 186 \mathrm{R}$ is frequent in African populations with minor allele frequencies ranging from $30 \%$ in South Africans [19] to between 25 and 51\% in other African populations [20]. The polymorphism is rare in white $(2-3 \%)$ and Asian $(0-10 \%)$ populations [20]. This A3G-H186R variant has been associated with higher viral loads, decreased $\mathrm{CD} 4^{+} \mathrm{T}$-cell counts and a more rapid progression to AIDS in patients homozygous for A3G-H186R [19,21-23]. However, other studies which mainly analysed the effect of the heterozygous $\mathrm{A} 3 \mathrm{G}^{\mathrm{WT} / \mathrm{H} 186 \mathrm{R}}$ variant found no association with disease progression [24-27]. Despite the clear correlation of A3G-H186R in vivo, the mechanism remains unknown $[21,28]$.

We recently showed that HIV-1 Vif adapts to different APOBEC3H (A3H) haplotypes in HIV-1-infected patients [29]. However, it remains unknown whether A3G haplotypes similarly select for specific Vif variants. If a given A3G variant is more or less susceptible to HIV-1 Vif mediated degradation, it is conceivable that this altered viral host interaction could result in an altered HIV/AIDS disease presentation.

Of note, most Vif-A3G studies have focused on subtype B Vif variants, which only represent $\sim 10 \%$ of all global infections [30]. However, the greatest burden of infections is in sub-Saharan Africa where HIV-1 subtype C predominates [30]. Only two studies, including one from our group, functionally analysed a limited number of subtype $\mathrm{C}$ variants. Both studies concluded that subtype C Vif had similar or enhanced activity against wild type $\mathrm{A} 3 \mathrm{G}$ as compared to subtype B Vifs. [31,32].

To our knowledge, the current study is the first to investigate the antiA3G phenotype of subtype C Vif alleles obtained from patients with distinct A3G haplotypes.

\section{Materials and methods}

\section{Study participants}

We selected 26 women from the 'Centre for the AIDS Programme of Research in South Africa' acute infection study (CAPRISA 002) in Durban, South Africa [33] based on the $A 3 G$ genotype information that was previously determined [19]. Plasma viremia and $\mathrm{CD} 4^{+}$ T-cell counts are regularly documented, and samples are stored for future research. Participants provided written informed consent, and ethical approval was obtained from the Biomedical Research Ethics Committee of the University of KwaZulu-Natal.

We obtained plasma samples from $11 \mathrm{~A} 3 \mathrm{G}^{\mathrm{WT} / \mathrm{WT}}, 10$ $\mathrm{A} 3 \mathrm{G}^{\mathrm{WT} / \mathrm{H} 186 \mathrm{R}}$ and $5 \mathrm{~A} 3 \mathrm{G}^{\mathrm{H} 186 \mathrm{R} / \mathrm{H} 186 \mathrm{R}}$ chronically infected, antiretroviral therapy-naïve study participants. The plasma viremia and absolute $\mathrm{CD} 4^{+}$T-cell counts were obtained approximately 36 months postinfection and were comparable between patients with different A3G haplotypes.

\section{HIV-1 Vif amplification and cloning}

Viral RNA was extracted from plasma using QIAamp Viral RNA Mini Kit (Qiagen, Hilden, Germany) and cDNA synthesized using Thermoscript RT PCR System (Invitrogen, ThermoFisher Scientific, Waltham, Massachusetts, USA). The HIV-1 Vif coding region was amplified by nested PCR with the Expand High Fidelity PCR System (Roche, Penzberg, Germany) using the primers Vif1-forward 5'AAAATTAGCAGGAAGAT GGCCAGT3' and Vif1-reverse $5^{\prime}$ CTCCGCTTCTTC CTGCCATAGGAGAT3', and Vif2-forward 5'TACTC TGGAAAGGTGAAGG3 ${ }^{\prime}$ and Vif2-reverse $5^{\prime}$ CTTCC TGCCATAGGAGATGCCTAA3'. Five separate PCR reactions were performed per patient and gel-purified amplicons were cloned into the pCR2.1 TOPO cloning vector (Invitrogen). Two to four clones of each PCR were sequenced resulting in 10-20 sequences for each patient. The GenBank accession numbers for the vif sequences generated in this study are KT881902-KT882293.

\section{Vif and A3G expression plasmids}

One representative HIV-1 Vif variant from each of the $11 \mathrm{~A} 3 \mathrm{G}^{\mathrm{WT} / \mathrm{WT}}$ and $5 \mathrm{~A} 3 \mathrm{G}^{\mathrm{H} 186 \mathrm{R} / \mathrm{H} 186 \mathrm{R}}$ donors was selected for functional characterization. The Vif ORF was carboxy-terminal FLAG tagged and cloned into the mammalian expression plasmid pCRV1, as previously described [34,35]. Carboxy-terminal haemagglutinintagged wild type A3G and A3G-H186R were cloned into the mammalian expression plasmid PTR600, as previously described [36].

\section{Cell culture}

TZM-bl cells were provided by J.C. Kappes and X. Wu through the AIDS Research and Reference Reagent Program, Division of AIDS, NIAID, National Institutes of Health, NIH Reagent Program. HEK-293T and 
TZM-bl were maintained at $37^{\circ} \mathrm{C}$ in a humidified atmosphere of $5 \% \mathrm{CO}_{2}$ in Dulbecco's high-glucose modified Eagle's medium (CellGro, Corning, New York, USA), supplemented with $10 \%$ foetal bovine serum and penicillin/streptomycin.

\section{A3G degradation and single-cycle viral infectivity assays}

HEK-293T cells were co-transfected with $500 \mathrm{ng}$ of HIV pNL4-3 $\Delta$ vif, $50 \mathrm{ng}$ of each Vif expression plasmid and $20 \mathrm{ng}$ of wild type or A3G-H186R with $4 \mathrm{mg} / \mathrm{ml}$ of polyethylenimine, as previously described [36]. The replication-competent molecular clone NL4-3 $\Delta$ Vif was provided by the AIDS Research and Reference Reagent Program, Division of AIDS, NIAID, National Institutes of Health. After $48 \mathrm{~h}$, viral supernatants were collected and the cells were lysed and analysed by western blot, as previously described [36]. Viral supernatants were used to infect TZM-bl cells, and $\beta$-galactosidase activity was measured $48 \mathrm{~h}$ postinfection, as previously described [29].

\section{Statistical analysis}

GraphPad Prism version 5.01 was used for statistical analyses (paired and unpaired $t$ tests). $P$ values less than 0.05 were considered significant. Average relative infectivity values and their SDs were calculated from representative triplicate transfections.

\section{Results}

\section{Phylogenetic analysis of Vif sequences}

Despite the availability of HIV-1 subtype $\mathrm{C}$ vif sequences [37-39], functional data regarding their antiA3G activity remains very limited [31,32]. We therefore cloned, sequenced and analysed HIV-1 subtype C vif alleles from patients homozygous for wild type A3G, homozygous for A3G-H186R and from heterozygous patients [19]. We generated 392 full length HIV-1 subtype C vif clonal sequences. Phylogenetic analysis confirmed that all sequences were subtype C (data not shown) and vif clonal sequences from each patient clustered independently (Fig. 1a). Intrapatient sequences differed between 0.1 and $4.9 \%$ and interpatient diversity ranged from 6.2 to $19.2 \%$ at the protein level. We observed no significant correlations between intrapatient sequence diversity and viral loads or $\mathrm{CD}^{+}$cell counts (data not shown).

An alignment of each patient's consensus sequences is shown in Fig. 1b. Putative sites of interaction with A3G or Cullin 5 and the E3 ubiquitin ligase complex are indicated by different colours (Fig. 1b). The sites of potential interaction with $\mathrm{A} 3 \mathrm{G}$ include amino acids at position 9, 22, 45 and 48 [34,40]; the YRHHY motif (40-44) [41]; the VHIPLx4-5Lx2YWGL motif (positions 55-59, 64, 69-72) [42]; tryptophans at (a)

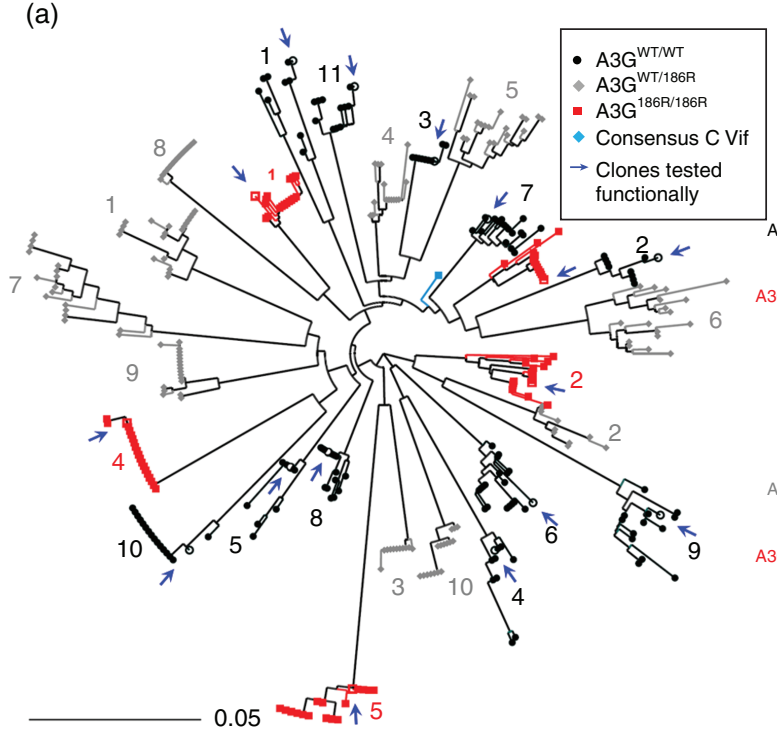

(b)

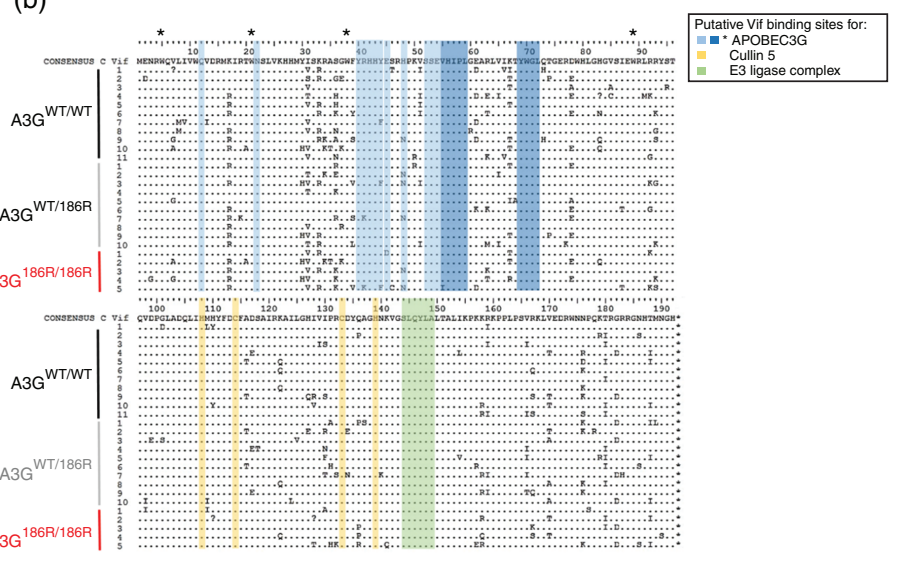

Fig. 1. Sequence analysis of patient-derived HIV-1 Vif sequences. (a) Neighbour joining phylogenetic tree of 392 full length HIV-1 vif clonal sequences shows HIV-1 vif clonal sequences from each of 26 participants forming independent clusters. The patient's A3G genotype from which Vif clones were derived are represented by the indicated symbols and colours. Vif clones that were functionally tested are represented by open symbols and arrows. Patient samples were assigned numbers that correspond in later figures. (b) Alignment of Vif amino acid consensus sequences of 26 study samples. Sequences are compared with a consensus subtype C reference sequence [obtained from the Los Alamos National Laboratory HIV database (http://www.hiv.lanl.gov)]. Protein domains putatively involved in interactions that lead to proteasomal degradation of A3G are indicated; blue indicates I9, N22, E45 and N48, YRHHY (40-44), amino acids 52-72, including the highlighted VHIPLx4-5Lx2YWGI motif which are important for binding to A3G. *Indicates tryptophan residues important for A3G binding; yellow indicates the HCCH motif important for binding to Cullin 5 and green shows SLQYLA motif important for recruitment of ubiquitin-ligase (E3) complex containing Elongin B and C, cullin-5 and Rbx. 
positions 5, 21, 38 and 89 [43], were not different between sequences. Similarly, the $\mathrm{HCCH}$ and SLQYLA motifs, which are binding sites of Cullin 5 [44,45] and Elongin C (144-149) were conserved [16,46,47].

\section{Phenotypic characterization of HIV subtype C Vif variants}

There is evidence that natural Vif variants differ in their ability to neutralize A3G [31,32,34], and we speculate that Vif diversity may emerge as it adapts to an individual's A3G repertoire. We functionally characterized the patient-derived subtype C Vif panel for A3G degradation and counteraction in single-cycle infectivity assays. We co-transfected HIV $\Delta$ Vif with individual Vif expression plasmids, wild type A3G or A3G-H186R. HIV-1 infectivity was subsequently analysed by infecting TZM-bl reporter cells with viral supernatants, collected 2 days posttransfection. Infectivity values were plotted relative to HIV-1 in the absence of A3G, which was set to $100 \%$.

We first looked at whether Vifs obtained from $\mathrm{A} 3 \mathrm{G}^{\mathrm{WT}}$ / WT or $A 3 G^{\mathrm{H} 186 \mathrm{R} / \mathrm{H} 186 \mathrm{R}}$ patients would differ in their ability to counteract A3G. All patient-derived Vif variants counteracted wild type A3G and A3G-H186R to similar levels, irrespective of the patient's A3G variant (Fig. 2, compare within a and $\mathrm{b}$, NS, unpaired $t$ test). This indicates that HIV-1 Vif does not adapt to the different A3G variants in vivo. Western blot analysis showed that the level of A3G degradation (compare with the no Vif control) was similar among Vifs (compare between Fig. 2a and $b$ ), which is in agreement with the infectivity data. Additionally, we observed that the expression levels of the individual Vif variants was highly variable, but was independent of both the patient's A3G genotype from which they were derived as well as of their activity to degrade and counteract A3G (Fig. 2a and b).

\section{Intrinsic antiviral activity of wild type A3G and A3G-H186R}

To determine whether the $\mathrm{A} 3 \mathrm{G}$ variants differ in the restriction activity, we compared viral infectivity in the presence of wild type A3G or A3G-H186H (compare between Fig. $2 a$ and $b$ ). The infectivity values in the presence of A3G-H186R were always significantly higher compared with wild type A3G $(P<0.0001$, paired $t$ test $)$, indicating that $\mathrm{A} 3 \mathrm{G}-\mathrm{H} 186 \mathrm{R}$ restricts HIV less efficiently than wild type A3G (Fig. 2c). This difference is likely attributable to the lower basal antiviral activity of A3GH186R, because the same result is also apparent in the absence of Vif (Fig. 2d, $4.32 \pm 0.21$ for wild type A3G versus $6.9 \pm 0.28$ for A3G-H186R, $P=0.0007$, unpaired $t$ test). This small but significant difference suggests that A3G-H186R intrinsically has reduced antiviral activity.

Taken together, we observed no evidence for functional adaptation of Vif to the different $\mathrm{A} 3 \mathrm{G}$ variants in vivo. Importantly, our data show that the wild type $A 3 G$ was more efficient at restricting HIV compared with A3GH186R variant, which may explain the accelerated HIV1 disease progression in $\mathrm{A}_{3} \mathrm{G}^{\mathrm{H} 186 \mathrm{R} / \mathrm{H} 186 \mathrm{R}}$ patients.

\section{Discussion}

Several studies showed that $\mathrm{A} 3 \mathrm{G}^{\mathrm{H} 186 \mathrm{R} / \mathrm{H} 186 \mathrm{R}}$ patients experience accelerated AIDS disease progression compared with individuals expressing $A 3 \mathrm{G}^{\mathrm{WT} / \mathrm{WT}}$ or (a)
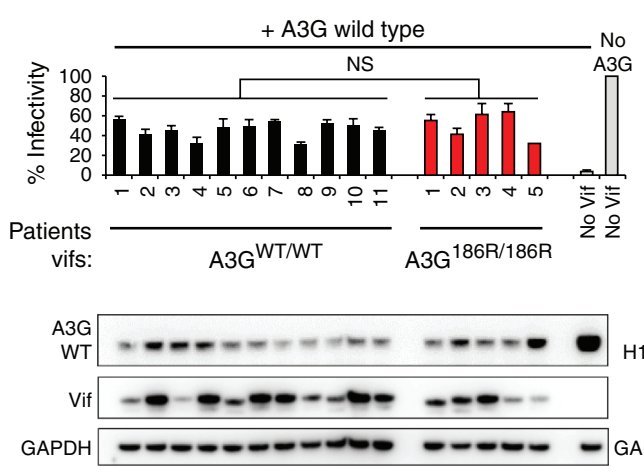

(b)

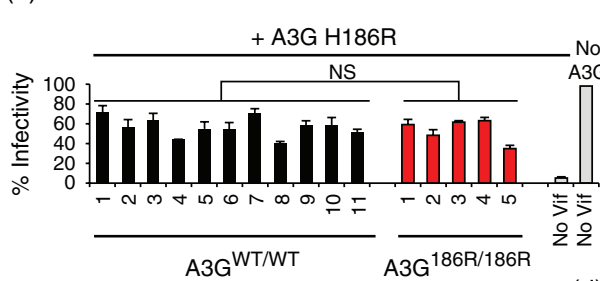

(c)

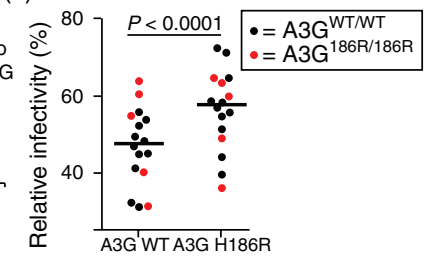

(d)
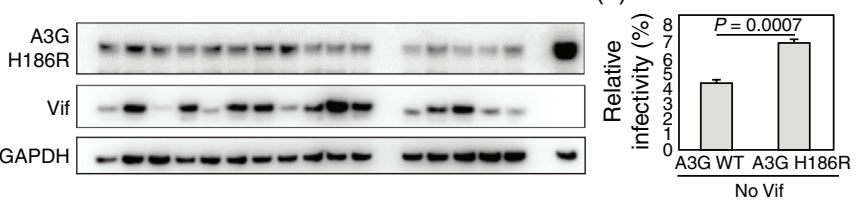

Fig. 2. Activity of patient-derived subtype $C$ Vifs against the wild type A3G and A3G-H186R variants. Patient-derived Vifs were cloned into an expression vector and co-transfected with NL4-3 $\Delta$ Vif and wild type A3G (a) or A3G-H186R (b) expression plasmids and infectivity of the produced viruses was tested on TZM-bl reporter cells. NS, unpaired $t$ test. 293T cell lysates were analysed by Western blot and probed for haemagglutinin, Vif and GAPDH serves as a loading control. Vif variants derived from homozygotes wild type A3G are represented by black bars, whereas Vifs derived from A3G-H186R homozygous carriers are represented by red bars. Error bars represent SDs from triplicate transfections. (c) Dot plot comparing the infectivity levels in the presence of wild type A3G or A3G-H186R, $P<0.0001$, paired $t$ test. (d) Comparison of infectivities in the presence of wild type A3G and A3G-H186R in the absence of $\mathrm{Vif}$ [also shown in (a)]. $P=0.0007$, unpaired $t$ test. Error bars represent SDs from triplicate transfections. 
A3G ${ }^{\text {WT/H186R }}[19,21-23]$. We observed that A3GH186R restricts HIV-1 less potently than wild type A3G, both in the presence as well as in the absence of Vif. This novel finding indicates that A3G-H186R intrinsically has less antiviral activity, which is in agreement with a previous study which used cell-free biochemical approaches which shows that A3G-H186R has reduced deaminase activity [48]. It is also possible that the H186R substitution may affect its packaging into virions, RNA binding or protein oligomerization, and further mechanistic studies are required to elucidate the reduced antiviral activity of A3G-H186R. We speculate that A3G is not always fully counteracted by Vif in vivo, and that the reduced restriction of A3G-H186R leads to higher viral loads and a more rapid disease progression in patients expressing $A 3 G^{\mathrm{H} 186 \mathrm{R} / \mathrm{H} 186 \mathrm{R}}$.

Studies show that SIV Vif adapts to polymorphisms in A3G $[4,49,50]$. We, therefore, anticipated that Vif would reduce its activity to degrade A3G-H186R because it poses less of a threat than $A 3 G$ wild type. However, Vif variants from $A 3 G^{\text {WT/WT }}$ or $A 3 G^{\mathrm{H} 186 \mathrm{R}}$; H186R showed no differences in activity, indicating that Vif does not adapt to A3G-H186R. It is conceivable that the difference in anti-HIV activity of the A3G variants is too small to create sufficient selective pressure for Vif adaptation, given that A3G-H186R has less antiviral activity than the wild type counterpart.

Together, polymorphisms in multiple APOBEC3 proteins such as $\mathrm{A} 3 \mathrm{G}$ and $\mathrm{A} 3 \mathrm{H}$ in combination with a HIV strain encoding a specific Vif variant could have profound effects on HIV replication and HIV-disease progression in patients $[19,21-23,28,29,51]$. Indeed, the time to progression to AIDS varies widely in patients, of which only a small percentage could be attributed to natural polymorphisms in genes, commonly associated with differential outcome such as CCR5 or HLA [52-58].

\section{Acknowledgements}

We thank all the CAPRISA 002 Acute Infection Study participants who are continuing to make an important contribution to HIV research. The scientific and supportive role of the whole CAPRISA 002 study and protocol teams is gratefully acknowledged. This study was funded in part by grants from South African Department of Science and Technology and National Research Foundation Research Chairs Initiative (grant no. 64809) (T.N.), the Victor Daitz Chair Foundation (T.N.), an International Early Career Scientist Award from the Howard Hughes Medical Institute (grant no. 55007427) (T.N.) and the NIH grants R01AI064001 (V.S.), R01 AI089246 (V.S.). The CAPRISA 002 study team has received support from the National Institute of Allergy and infectious Disease (NIAID), National Institutes of Health (NIH) (grants nos. AI51794, AI104387, AI115981, AI116086), from CONRAD (USAID cooperative grant no. GP00-08-00005-00, subproject agreement no. PPA-09-046), from the National Research Foundation (grant no. 67385), the Technology Innovation Agency, and the Columbia University-Southern African Fogarty AIDS International Training and Research Programme (AITRP) funded by the Fogarty International Center, NIH (grant no. D43TW00231).

Author contributions: K.R., M.O., V.S., T.N. conceived and designed the experiments. K.R., M.O. and M.L. performed the experiments. K.R. and M.O. analysed the data. N.G. heads and manages the study cohort and provided the samples. All the authors contributed to the manuscript writing and reviewed the final version.

\section{Conflicts of interest}

There are no conflicts of interest.

\section{References}

1. Harris RS, Hultquist JF, Evans DT. The restriction factors of human immunodeficiency virus. / Biol Chem 2012; 287:4087540883.

2. Kirchhoff F. Immune evasion and counteraction of restriction factors by HIV-1 and other primate lentiviruses. Cell Host Microbe 2010; 8:55-67.

3. Malim MH, Bieniasz PD. HIV restriction factors and mechanisms of evasion. Cold Spring Harbor Perspect Med 2012; 2:a006940.

4. Malim MH, Emerman M. HIV-1 accessory proteins - ensuring viral survival in a hostile environment. Cell Host Microbe 2008; 3:388-398.

5. O'Brien SJ, Nelson GW. Human genes that limit AIDS. Nat Genet 2004; 36:565-574.

6. Sharp PM, Hahn BH. Origins of HIV and the AIDS pandemic. Cold Spring Harbor Perspect Med 2011; 1:a006841.

7. Simon V, Bloch N, Landau NR. Intrinsic host restrictions to HIV-1 and mechanisms of viral escape. Nat Immunol 2015; 16:546-553.

8. Mangeat B, Turelli P, Caron G, Friedli M, Perrin L, Trono D. Broad antiretroviral defence by human APOBEC3G through lethal editing of nascent reverse transcripts. Nature 2003; 424:99-103.

9. Sheehy AM, Gaddis NC, Choi JD, Malim MH. Isolation of a human gene that inhibits HIV-1 infection and is suppressed by the viral Vif protein. Nature 2002; 418:646-650.

10. Harris RS, Bishop KN, Sheehy AM, Craig HM, Petersen-Mahrt SK, Watt IN, et al. DNA deamination mediates innate immunity to retroviral infection. Cell 2003; 113:803-809.

11. Zhang H, Yang B, Pomerantz RJ, Zhang C, Arunachalam SC, Gao $L$. The cytidine deaminase CEM15 induces hypermutation in newly synthesized HIV-1 DNA. Nature 2003; 424:94-98.

12. Guo F, Cen S, Niu M, Yang Y, Gorelick RJ, Kleiman L. The interaction of APOBEC $3 G$ with human immunodeficiency virus type 1 nucleocapsid inhibits tRNA3Lys annealing to viral RNA. J Virol 2007; 81:11322-11331.

13. Klarmann GJ, Chen X, North TW, Preston BD. Incorporation of uracil into minus strand DNA affects the specificity of plus strand synthesis initiation during lentiviral reverse transcription. J Biol Chem 2003; 278:7902-7909.

14. Newman EN, Holmes RK, Craig HM, Klein KC, Lingappa JR, Malim $\mathrm{MH}$, et al. Antiviral function of APOBEC3G can be dissociated from cytidine deaminase activity. Curr Biol 2005; 15:166-170. 
15. Shindo K, Takaori-Kondo A, Kobayashi M, Abudu A, Fukunaga $\mathrm{K}$, Uchiyama T. The enzymatic activity of CEM15/Apobec-3G is essential for the regulation of the infectivity of HIV-1 virion but not a sole determinant of its antiviral activity. I Biol Chem 2003; 278:44412-44416.

16. Marin M, Rose KM, Kozak SL, Kabat D. HIV-1 Vif protein binds the editing enzyme APOBEC $3 G$ and induces its degradation. Nat Med 2003; 9:1398-1403.

17. Sheehy AM, Gaddis NC, Malim MH. The antiretroviral enzyme APOBEC3G is degraded by the proteasome in response to HIV1 Vif. Nat Med 2003; 9:1404-1407.

18. Stopak K, de Noronha C, Yonemoto W, Greene WC. HIV-1 Vif blocks the antiviral activity of APOBEC 3G by impairing both its translation and intracellular stability. Mol Cell 2003; 12:591601.

19. Reddy K, Winkler CA, Werner L, Mlisana K, Abdool Karim SS Ndung'u T. APOBEC $3 G$ expression is dysregulated in primary HIV-1 infection and polymorphic variants influence CD4+ Tcell counts and plasma viral load. AIDS 2010; 24:195-204.

20. Genomes Project C, Abecasis GR, Auton A, Brooks LD, DePristo $M A$, Durbin $R M$, et al. An integrated map of genetic variation from 1092 human genomes. Nature 2012; 491:56-65.

21. An $P$, Bleiber $G$, Duggal $P$, Nelson G, May M, Mangeat $B$, et al. APOBEC3G genetic variants and their influence on the progression to AIDS. I Virol 2004; 78:11070-11076.

22. Bunupuradah $T$, Imahashi $M$, lampornsin $T$, Matsuoka $K$ Iwatani $\mathrm{Y}$, Puthanakit $\mathrm{T}$, et al. Association of APOBEC3G genotypes and CD4 decline in Thai and Cambodian HIVinfected children with moderate immune deficiency. AIDS Res Ther 2012; 9:34.

23. Singh KK, Wang Y, Gray KP, Farhad M, Brummel S, Fenton T, et al. Genetic variants in the host restriction factor APOBEC3G are associated with HIV-1-related disease progression and central nervous system impairment in children. J Acquir Immune Defic Syndr 2013; 62:197-203.

24. Bizinoto MC, Leal E, Diaz RS, Janini LM. Loci polymorphisms of the APOBEC 3G gene in HIV type 1 -infected Brazilians. AIDS Res Hum Retroviruses 2011; 27:137-141.

25. De Maio FA, Rocco CA, Aulicino PC, Bologna R, Mangano A, Sen L. Effect of HIV-1 Vif variability on progression to pediatric AIDS and its association with APOBEC3G and CUL5 polymorphisms. Infect Genet Evol 2011; 11:1256-1262.

26. De Maio FA, Rocco CA, Aulicino PC, Bologna R, Mangano A, Sen L. APOBEC3-mediated editing in HIV type 1 from pediatric patients and its association with APOBEC3G/CUL5 polymorphisms and Vif variability. AIDS Res Hum Retroviruses 2012; 28:619-627.

27. Do H, Vasilescu A, Diop G, Hirtzig T, Heath SC, Coulonges C, et al. Exhaustive genotyping of the CEM15 (APOBEC3G) gene and absence of association with AIDS progression in a French cohort. I Infect Dis 2005; 191:159-163.

28. Duggal NK, Fu W, Akey JM, Emerman M. Identification and antiviral activity of common polymorphisms in the APOBEC 3 locus in human populations. Virology 2013; 443:329-337.

29. Ooms M, Brayton B, Letko M, Maio SM, Pilcher CD, Hecht FM, et al. HIV-1 Vif adaptation to human APOBEC3H haplotypes. Cell Host Microbe 2013; 14:411-421.

30. Hemelaar J, Gouws E, Ghys PD, Osmanov S, Isolation W$\mathrm{UNfH}$, Characterisation. Global trends in molecular epidemiology of HIV-1 during 2000-2007. AIDS 2011; 25:679-689.

31. Binka $M$, Ooms $M$, Steward $M$, Simon V. The activity spectrum of Vif from multiple HIV-1 subtypes against APOBEC3G, APOBEC3F, and APOBEC3H. / Virol 2012; 86:49-59.

32. Iwabu $Y$, Kinomoto $M$, Tatsumi $M$, Fujita H, Shimura M, Tanaka $Y$, et al. Differential anti-APOBEC3G activity of HIV-1 Vif proteins derived from different subtypes. I Biol Chem 2010; 285:35350-35358.

33. van Loggerenberg F, Mlisana K, Williamson C, Auld SC, Morris L, Gray CM, et al. Establishing a cohort at high risk of HIV infection in South Africa: challenges and experiences of the CAPRISA 002 acute infection study. PLoS One 2008; 3:e1954.

34. Simon V, Zennou V, Murray D, Huang Y, Ho DD, Bieniasz PD. Natural variation in Vif: differential impact on APOBEC3G/3F and a potential role in HIV-1 diversification. PLOS Pathog 2005; 1:e6.

35. Zennou V, Perez-Caballero D, Gottlinger H, Bieniasz PD. APOBEC3G incorporation into human immunodeficiency virus type 1 particles. J Virol 2004; 78:12058-12061.
36. Letko M, Silvestri G, Hahn BH, Bibollet-Ruche F, Gokcumen $\mathrm{O}$ Simon $\mathrm{V}$, et al. Vif proteins from diverse primate lentiviral lineages use the same binding site in APOBEC3G. / Virol 2013; 87:11861-11871.

37. Jacobs GB, Nistal M, Laten A, van Rensburg EJ, Rethwilm A Preiser W, et al. Molecular analysis of HIV type 1 vif sequences from Cape Town, South Africa. AIDS Res Hum Retroviruses 2008; 24:991-994.

38. Scriba TJ, Treurnicht FK, Zeier M, Engelbrecht S, van Rensburg EJ. Characterization and phylogenetic analysis of South African HIV-1 subtype C accessory genes. AIDS Res Hum Retroviruses 2001; 17:775-781.

39. Bell CM, Connell BJ, Capovilla A, Venter WD, Stevens WS Papathanasopoulos MA. Molecular characterization of the HIV type 1 subtype $\mathbf{C}$ accessory genes vif, vpr, and vpu. AIDS Res Hum Retroviruses 2007; 23:322-330.

40. Wichroski MJ, Ichiyama K, Rana TM. Analysis of HIV-1 viral infectivity factor-mediated proteasome-dependent depletion of APOBEC3G: correlating function and subcellular localization. I Biol Chem 2005; 280:8387-8396.

41. Russell RA, Pathak VK. Identification of two distinct human immunodeficiency virus type 1 Vif determinants critical for interactions with human APOBEC3G and APOBEC3F. / Virol 2007; 81:8201-8210.

42. He Z, Zhang W, Chen G, Xu R, Yu XF. Characterization of conserved motifs in HIV-1 Vif required for APOBEC3G and APOBEC3F interaction. / Mol Biol 2008; 381:10001011.

43. Tian $C, Y u$ X, Zhang $W$, Wang $T, X u$ R, Yu XF. Differential requirement for conserved tryptophans in human immunodeficiency virus type 1 Vif for the selective suppression of APOBEC3G and APOBEC3F. / Virol 2006; 80:3112-3115.

44. Luo K, Xiao Z, Ehrlich E, Yu Y, Liu B, Zheng S, et al. Primate lentiviral virion infectivity factors are substrate receptors that assemble with cullin 5-E3 ligase through a $\mathrm{HCCH}$ motif to suppress APOBEC3G. Proc Natl Acad Sci USA 2005; 102:11444-11449.

45. Xiao Z, Xiong Y, Zhang W, Tan L, Ehrlich E, Guo D, et al. Characterization of a novel Cullin5 binding domain in HIV-1 Vif. J Mol Biol 2007; 373:541-550.

46. Mehle A, Strack B, Ancuta P, Zhang C, McPike M, Gabuzda D. Vif overcomes the innate antiviral activity of APOBEC 3G by promoting its degradation in the ubiquitin-proteasome pathway. I Biol Chem 2004; 279:7792-7798.

47. Yu X, Yu Y, Liu B, Luo K, Kong W, Mao P, et al. Induction of APOBEC3G ubiquitination and degradation by an HIV-1 Vif-Cul5-SCF complex. Science 2003; 302:10561060.

48. Feng $Y$, Chelico L. Intensity of deoxycytidine deamination of HIV-1 proviral DNA by the retroviral restriction factor APOBEC3G is mediated by the noncatalytic domain. I Biol Chem 2011; 286:11415-11426.

49. Compton AA, Hirsch VM, Emerman $M$. The host restriction factor APOBEC $3 G$ and retroviral Vif protein coevolve due to ongoing genetic conflict. Cell Host Microbe 2012; 11:9198.

50. Krupp A, McCarthy KR, Ooms M, Letko M, Morgan JS, Simon $\mathrm{V}$, et al. APOBEC3G polymorphism as a selective barrier to cross-species transmission and emergence of pathogenic SIV and AIDS in a primate host. PLoS Pathog 2013; 9:e1003641.

51. Refsland EW, Hultquist JF, Luengas EM, Ikeda T, Shaban NM, Law EK, et al. Natural polymorphisms in human APOBEC3H and HIV-1 Vif combine in primary $\mathrm{T}$ lymphocytes to affect viral G-to-A mutation levels and infectivity. PLOS Genet 2014; 10:e1004761.

52. Carrington $M, O^{\prime} B r i e n ~ S J$. The influence of HLA genotype on AIDS. Annu Rev Med 2003; 54:535-551.

53. Dean $M$, Carrington $M$, Winkler C, Huttley GA, Smith MW, Allikmets R, et al. Genetic restriction of HIV-1 infection and progression to AIDS by a deletion allele of the CKR5 structural gene. Hemophilia Growth and Development Study, Multicenter AIDS Cohort Study, Multicenter Hemophilia Cohort Study, San Francisco City Cohort, ALIVE Study. Science 1996; 273:1856-1862.

54. Kawashima $Y$, Pfafferott K, Frater I, Matthews P, Payne R, Addo $M$, et al. Adaptation of HIV-1 to human leukocyte antigen class I. Nature 2009; 458:641-645. 
55. Leslie AJ, Pfafferott KJ, Chetty P, Draenert R, Addo MM, Feeney $M$, et al. HIV evolution: CTL escape mutation and reversion after transmission. Nat Med 2004; 10:282-289.

56. Liu R, Paxton WA, Choe S, Ceradini D, Martin SR, Horuk R, et al. Homozygous defect in HIV-1 coreceptor accounts for resistance of some multiply-exposed individuals to HIV-1 infection. Cell 1996; 86:367-377.
57. Rousseau CM, Daniels MG, Carlson JM, Kadie C, Crawford $H$, Prendergast $A$, et al. HLA class I-driven evolution of human immunodeficiency virus type 1 subtype c proteome: immune escape and viral load. J Virol 2008; 82:64346446.

58. Stephens HA. HIV-1 diversity versus HLA class I polymorphism. Trends Immunol 2005; 26:41-47. 